\title{
Matrix metalloproteinase 2 knockdown suppresses the proliferation of HepG2 and Huh7 cells and enhances the cisplatin effect
}

https://doi.org/10.1515/med-2019-0039

received December 19, 2018; accepted April 18, 2019

\begin{abstract}
Background. This study evaluated the functions of matrix metalloproteinase 2 (MMP2) in hepatocellular carcinoma (HCC) cells and assessed the effects of MMP2 on HCC cell sensitivity to cisplatin.
\end{abstract}

Methodology. HepG2 and Huh7 cells were cultured. A pre-experiment was performed to explore the optimal transduction conditions of the MMP2-siRNA lentivirus (si-MMP2). Quantitative real-time PCR and western blot assays were performed to measure the expression levels of MMP2 in HepG2 and Huh7 cells. An MTT assay was used to evaluate cell proliferation, and flow cytometry analysis was applied to examine cell apoptosis. A Transwell assay was carried out to assess cell invasion.

Results. The optimal virus:cell ratio was 100 multiplicity of infection (MOI) for both cells, and the optimal transduction times for HepG2 and Huh7 cells were $48 \mathrm{~h}$ and 72 $\mathrm{h}$, respectively. MMP2 knockdown significantly decreased the mRNA and protein levels of MMP2 in both cell lines $(\mathrm{P}<0.01)$. MMP2 knockdown significantly decreased the proliferation and increased the apoptosis of HepG2 and Huh7 cells $(\mathrm{P}<0.01)$. Co-treatment with si-MMP2 and cisplatin significantly increased the sensitivity of HepG2 and Huh7 cells to cisplatin $(\mathrm{P}<0.01)$.

Conclusion. MMP2 may act as an oncogene and may be a potential therapeutic target in HCC.

Keywords: Matrix metalloproteinase 2 (MMP2); Hepatocellular carcinoma; Proliferation; Apoptosis; Cisplatin

\footnotetext{
*Corresponding author: Jianzhao Huang, Department of Hepatobiliary Surgery, Guizhou Provincial People's Hospital, No. 1 Baoshan South Road, Guizhou 550002, China, Tel: 86-13765809981, Email: huangjzgz@126.com

Jiangwei Liu, Xiaocheng Li, Yan Liu, Department of Hepatobiliary Surgery, Guizhou Provincial People’s Hospital, Guizhou 550002, China
}

\section{Introduction}

Hepatocellular carcinoma (HCC) is the main type of liver cancer, accounting for more than half of all liver cancer cases $[1,2]$. Currently, the therapeutic options for HCC patients are poor and mainly include surgical resection, liver transplantation, and several antitumour drugs [3-5]. However, the therapeutic effects are extremely limited.

The matrix metalloproteinases (MMPs), consisting of a series of subtypes in humans, are zinc-dependent endopeptidases that are involved in the digestion of the extracellular matrix (ECM) during cell invasion or migration [6, 7]. To date, more than 22 human MMPs and 25 vertebrate homologues have been identified [8]. MMPs are produced as inactive zymogens and are maintained in the inactive form by the interaction between a cysteine in the propeptide domain and a zinc ion in the catalytic domain [7]. Activated MMPs have been demonstrated to be associated with various cell physiological processes, including cell apoptosis, proliferation, invasion, and migration $[9,10]$. Therefore, MMP activation is considered to play a critical role in the progression of human cancers. Recently, MMP2 was reported to participate in the tumourigenesis of multiple tumours, such as thyroid cancer, lung cancer, and ovarian cancer [11-13]. However, whether MMP2 is involved in the tumourigenesis of HCC remains largely unclear.

This study aimed to evaluate the underlying functions of MMP2 in HCC cells and to assess the effects of MMP2 on the sensitivity of HCC cells to cisplatin. We first synthesized an effective siRNA against MMP2 (si-MMP2), and two HCC cell lines (HepG2 and Huh7) were transduced with si-MMP2-carrying lentivirus to block MMP2 expression. In subsequent functional assays, we found that the knockdown of MMP2 significantly inhibited cell proliferation and invasion and promoted cell apoptosis, suggesting that MMP2 may act as an oncogene in HCC. In addition, we found that the knockdown of MMP2 in HCC cells enhanced the sensitivity of HepG2 and Huh7 cells to cisplatin, indicating that MMP2 inhibitors may serve as enhancers of antitumour drugs. 


\section{Materials and methods}

\subsection{Cell lines and culture}

The HCC cell lines HepG2 and Huh7 were both purchased from the Shanghai Institutes for Biological Sciences, Chinese Academy of Sciences, and maintained in a humidified cell incubator containing $5 \% \mathrm{CO}_{2}$ and $95 \%$ air at $37^{\circ} \mathrm{C}$ with RPMI 1640 medium supplemented with 100 $\mathrm{U} / \mathrm{ml}$ penicillin, $100 \mathrm{U} / \mathrm{ml}$ streptomycin and $10 \%$ foetal bovine serum (FBS).

\subsection{Experiments on optimal transduction conditions}

The optimal transduction concentrations and times were determined using western blot assays. Three parallel experiments were used in each treatment.

Gene silencing was performed using a lentivirus-mediated method [14]. The negative control siRNA (non-silencing siRNA with a scrambled sequence; si-con) lentivirus and the MMP2-siRNA (si-MMP2; target sequence, AGU AGA UCC AGU AUU CAU UCC CUG C [15]) lentivirus were purchased from Sangon Biotech, Shanghai, China. Polybrene (Life Technologies; Carlsbad, CA, US) was used as the transduction enhancer. The lentivirus transduction procedures were performed according to the instructions indicated by the manufacturer (Sangon Biotech, China).

\subsubsection{Optimal transduction concentration}

HepG2 and Huh7 cells were seeded into 24-well plates at a density of $1 \times 10^{5}$ cells/well. A centrifuge tube $(1.5 \mathrm{ml})$ was used, and serum-free culture medium without antibiotics at $150 \mu \mathrm{l}$ was added. Afterwards, $15 \mu \mathrm{l}$ of polybrene was added. The mixtures were applied to the cells to constitute the normal control, polybrene, si-con, 90 multiplicity of infection (MOI; lentivirus-cell ratio), $100 \mathrm{MOI}$, and 110 MOI groups. After $6 \mathrm{~h}$ of transduction, the complex culture medium was replaced with fresh medium. Then, the cells were incubated in $5 \% \mathrm{CO}_{2}$ and saturated humidity at $37^{\circ} \mathrm{C}$ for $72 \mathrm{~h}$.

\subsubsection{Optimal transduction time}

HepG2 and Huh7 cells were seeded at a density of $1 \times 10^{5}$ cells/well. Serum-free culture medium without antibiotics at $300 \mu \mathrm{l}$ was added to a $1.5 \mathrm{ml}$ centrifuge tube. Polybrene (15 $\mu \mathrm{l})$ was added. si-MMP2 was applied at the optimal concentration. Afterwards, the mixtures were applied to the cells to constitute the normal control, polybrene, 12-h, 24-h, 48-h and 72-h groups according to different transduction times. Other culture conditions were the same as the conditions mentioned above. An si-control group at the same concentration as that of si-MMP2 was also used for the 72-h culture.

\subsubsection{Western blot}

Total proteins of the treated HCC cells were extracted using RIPA buffer (50 mM Tris, $150 \mathrm{mM} \mathrm{NaCl}, 1 \%$ NP-40, $1 \%$ sodium deoxycholate, and $0.05 \%$ SDS, $\mathrm{pH}$ 7.4). After centrifugation by a refrigerated centrifuge (Eppendorf, TGL-18R) at $12,000 \mathrm{rpm} / \mathrm{min}$ at $4^{\circ} \mathrm{C}$ for $15 \mathrm{~min}$, the supernatants were collected, and a protein assay reagent (Bio-Rad Laboratory, Hercules, CA, USA) was applied to determine the protein concentration. A total of $50 \mu \mathrm{g}$ of protein was electrophoresed on a 10\% SDS-PAGE gel and transferred onto PVDF membranes. After blocking with 5\% non-fat dried milk at room temperature for $2 \mathrm{~h}$, membranes were incubated with primary antibodies against MMP2 (mouse, 1:1,000, Ab86607, Abcam) and actin (mouse, 1:2000, Santa Cruz Biotechnology, USA) at $4^{\circ} \mathrm{C}$ overnight. The membranes were washed thrice for $15 \mathrm{~min}$ for each wash with Tris-buffered saline with Tween 20 (TBS-T; containing $1.65 \mathrm{ml}$ of $20 \%$ Tween 20 and $700 \mathrm{ml}$ of TBS). Then, the membranes were incubated with horseradish peroxidase-conjugated donkey anti-mouse IgG (1:3,000; Proteintech; Rosemont, IL, USA) for 45-60 min. After extensive washing, the blots were developed by ECL (Thermo Fisher Scientific Inc.; Waltham, MA, USA). Chemiluminescence was captured on a UVP BioImaging system (UVP; Cambridge, UK).

\subsection{MMP2 knockdown experiments}

Western blot assays showed that the optimal virus-cell ratio was $100 \mathrm{MOI}$ for both cells and that the optimal transduction time for HepG2 and Huh7 cells was $48 \mathrm{~h}$ and 72 h, respectively (Supplementary Figures 1 and 2).

In the following experiments, the obtained optimal transduction conditions were used, with six parallel wells for each group. 

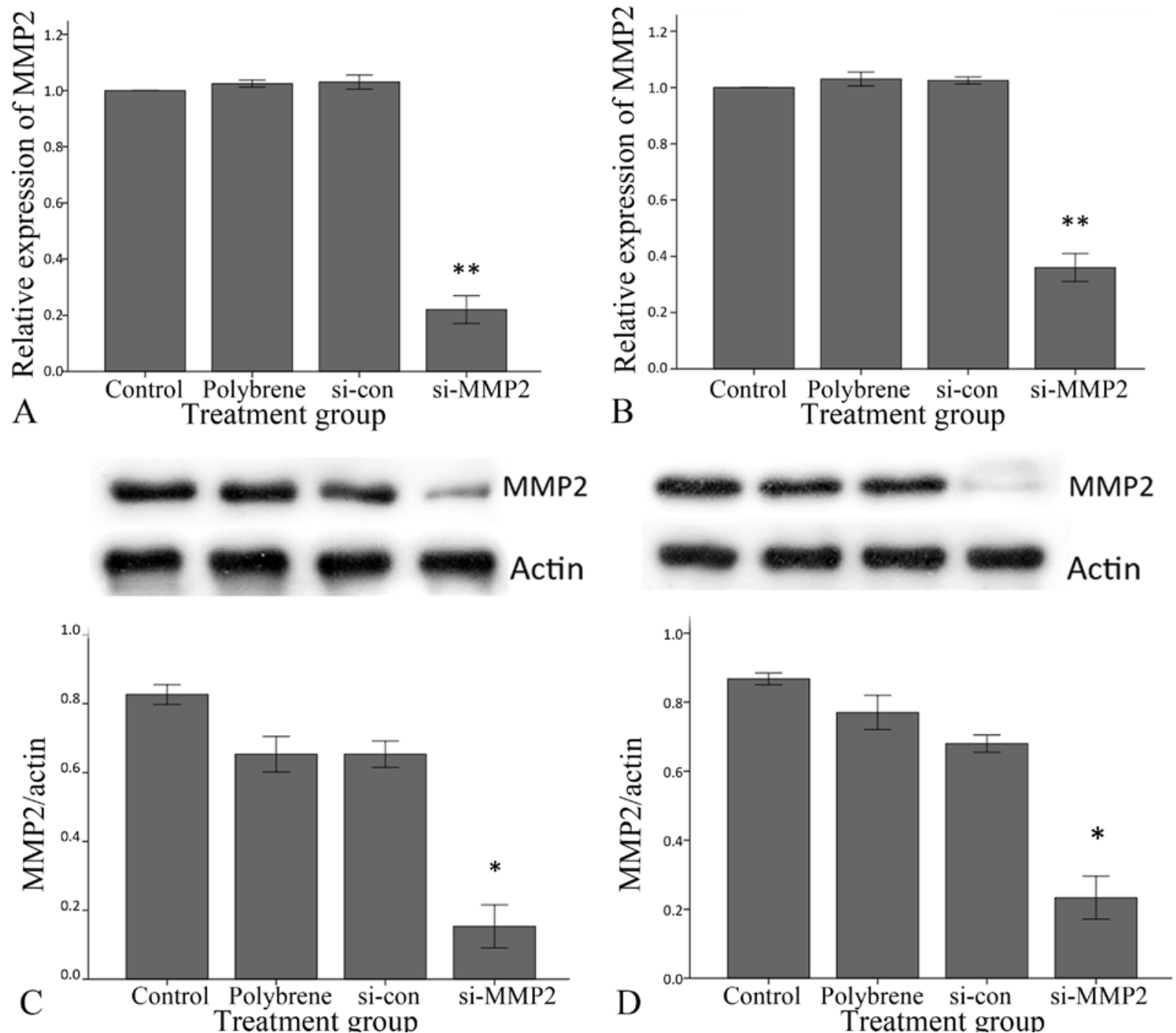

Figure 1. si-MMP2 knockdown efficiency. A, The knockdown efficiency according to the relative mRNA expression levels of MMP2 in HepG2 cells in the normal control, polybrene, si-control, and si-MMP2 groups based on real-time quantitative polymerase chain reaction (RT-qPCR). $B$, The knockdown efficiency according to the relative mRNA expression levels of MMP2 in Huh7 cells in the normal control, polybrene, si-control, and si-MMP2 groups based on RT-qPCR. C, The relative protein levels of MMP2 in HepG2 cells in the normal control, polybrene, si-control, and si-MMP2 groups based on western blot assays. D, The relative protein levels of MMP2 in Huh7 cells in the normal control, polybrene, si-control, and si-MMP2 groups based on western blot assays. ${ }^{*} \mathrm{P}<0.01$ vs. the normal control group, according to one-factor analysis of variance (ANOVA) followed by a post hoc least significant difference (LSD) test.

\subsubsection{Validation of knockdown efficiency}

HepG2 and Huh7 cells were seeded into 24-well plates at a concentration of $1 \times 10^{5}$ cells/well and incubated at $37^{\circ} \mathrm{C}$ overnight. Transduction was performed according to the optimal transduction conditions obtained in this study. The knockdown efficiency was validated by real-time quantitative polymerase chain reaction (RT-qPCR) and western blot assays [15].

\subsubsection{RT-qPCR}

Total RNAs of treated HepG2 and Huh7 cells were prepared using TRIzol reagent (Invitrogen, Carlsbad, CA, USA) following the protocol from the manufacturer. cDNA was synthesized using a Revert Aid First Strand cDNA Synthesis Kit (Thermo; USA) based on the total RNAs extracted from treated HCC cells. qRT-PCR was conducted on a 7900 HT Fast system (Applied Biosystems, California, USA) with the following protocol: $94^{\circ} \mathrm{C}, 5 \mathrm{~min}$; 40 cycles of amplification $\left(94^{\circ} \mathrm{C}, 30 \mathrm{~s}\right.$ and $\left.62^{\circ} \mathrm{C}, 40 \mathrm{~s}\right)$; and then $72^{\circ} \mathrm{C}$, $10 \mathrm{~min}$. The sequences of the MMP2 primers were 5'-AGACAGTGGATGATGCCTTTGC-3' (forward) and 5'-GGAGTC- 


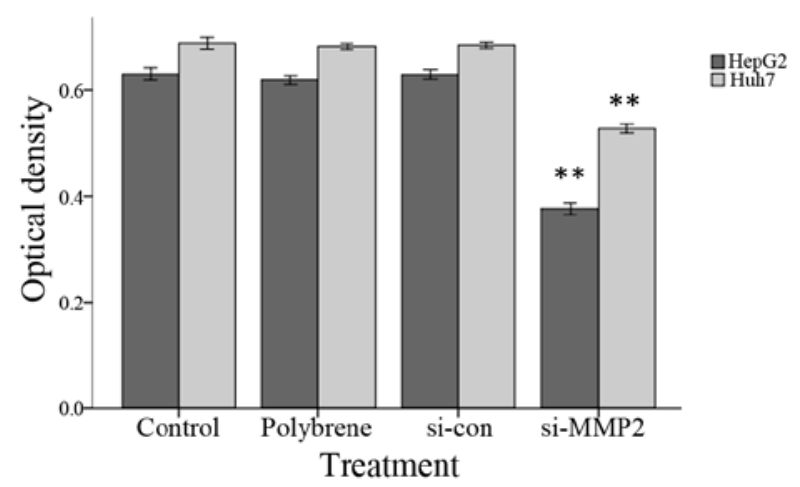

Figure 2: Effects of MMP2 knockdown on cell proliferation of HepG2 (at $48 \mathrm{~h}$ after transduction) and Huh7 cells (at $72 \mathrm{~h}$ after transduction) using MTT assays. ${ }^{* \star} P<0.01$ vs. the normal control group according to one-factor analysis of variance (ANOVA) followed by a post hoc least significant difference (LSD) test.

CGTCCTTACCGTCAAA-3' (reverse), and those of actin were 5'-CATCCTGCGTCTGGACCTGG-3' (forward) and 5'-TAATGTCACGCACGATTTCC-3' (reverse). The results were analysed using the $2^{-\Delta \Delta C t}$ method [16], where $\Delta \mathrm{Ct}=\Delta \mathrm{Ct}$ of the target or that of the reference (actin), and $-\Delta \Delta \mathrm{Ct}=$ average $\Delta \mathrm{Ct}$ of the control or that of the target. The $2^{-\Delta \Delta C t}$ method reflects the relative expression of the target gene to that of the control.

\subsubsection{Western blot}

The procedures were performed as described above.

\subsubsection{Cell invasion assay (Transwell assay)}

The cell invasive ability of treated HCC cells was determined using Transwell chambers $(8-\mu \mathrm{m}$ pore size; BD Biosciences, San Jose, CA, USA) with Matrigel coating (BD Biosciences). The upper chamber was filled with 200 $\mu \mathrm{l}$ of treated HepG2 and Huh7 cells $\left(1 \times 10^{5}\right)$ in serum-free medium, and $500 \mu \mathrm{l}$ of medium containing $5 \%$ FBS was added into the lower chamber. After incubating at $37^{\circ} \mathrm{C}$ for $24 \mathrm{~h}$, the non-invasive cells were removed, and the invasive cells were fixed and stained using crystal violet. Invasive cell numbers were counted under a microscope $(\times 100)$. Five visual fields were randomly selected, and the average was obtained.

\subsubsection{MTT assays}

Cell viability was assessed by MTT assays following the procedures described in the literature [17]. In this study,
HepG2 and Huh7 cells were infected according to the optimal transduction conditions obtained in this study. The optimal virus:cell ratios for both cell lines were 100 MOI, and the optimal transduction times for HepG2 and Huh7 cells were 48 and $72 \mathrm{~h}$, respectively. Then, the transduced cells were transferred to a 96-well plate for MTT assays at a density of $2 \times 10^{4}$ cells/well. After washing three times with phosphate buffered saline (PBS), cells were incubated with $20 \mu \mathrm{l}$ of MTT solution at $5 \mathrm{mg} / \mathrm{ml}$ for $4 \mathrm{~h}$ at $37^{\circ} \mathrm{C}$ in a humidified $\mathrm{CO}_{2}$ incubator. The media were removed, and then $150 \mu \mathrm{l}$ dimethylsulphoxide (DMSO) was added into each well and incubated at room temperature for $10 \mathrm{~min}$. Finally, the absorbance was measured at $490 \mathrm{~nm}$ with a microplate reader (Sunrise ${ }^{\mathrm{TM}}$; Tecan Group Ltd., Switzerland).

\subsubsection{Apoptosis analysis}

The cell apoptosis rate of HCC cells infected with si-MMP2 lentivirus was determined by Annexin-V/PI double staining followed by flow cytometry analysis [18]. Briefly, treated HepG2 and Huh7 cells were seeded in 24-well plates at a concentration of $2 \times 10^{4}$ cells/well and maintained overnight at $37^{\circ} \mathrm{C}$. After washing with cold PBS, HepG2 and Huh7 cells were resuspended in $500 \mu \mathrm{l}$ binding buffer containing $5 \mu \mathrm{l}$ PI and $5 \mu \mathrm{l}$ FITC-labelled Annexin-V and incubated for $10 \mathrm{~min}$ in the dark. Subsequently, apoptotic HepG2 and Huh7 cells were analysed with a FACScan flow cytometer and Kaluza analysis software (Beckman, Fullerton, CA, USA).

\subsubsection{Cisplatin cytotoxicity assays}

HepG2 and Huh7 cells were infected according to the optimal transduction conditions and were seeded in a 96-well plate at a density of $2 \times 10^{4}$ cells/well. Cisplatin was added into each well, and the final concentration of cisplatin was $50 \mu \mathrm{g} / \mathrm{ml}$. The incubation with cisplatin was conducted following a procedure described in the literature [19]. At $24 \mathrm{~h}$ after incubation, MTT assays were used to examine the cell viability of HCC cells, and the procedures were performed as described previously.

\subsection{Statistical analysis}

All the data are expressed as the mean \pm standard deviation and processed with SPSS 24.0 software (SPSS, Inc., Chicago, USA). The normality of the data distribution was 
tested using the Kolmogorov-Smirnov method, and the homogeneity of variance was determined using Levene's test. One-factor analysis of variance (ANOVA) followed by a post hoc least significant difference (LSD) test was performed for comparisons between groups. $\mathrm{P}<0.05$ and $\mathrm{P}<0.01$ were considered significant and very significant, respectively.

\section{Results}

\subsection{Knockdown efficiency of si-MMP2}

The knockdown efficiency of si-MMP2 was evaluated using RT-qPCR and western blot assays. No significant differences in the mRNA expression of MMP2 were observed among the control, polybrene, and si-con groups in both cell lines $(\mathrm{P}>0.05)$; however, compared with that in any other group in the same cell line, the MMP2 mRNA expression in the si-MMP2 group was significantly downregulated, indicating a significantly increased MMP2 knockdown efficiency $(\mathrm{P}<0.01$; Figure $1 \mathrm{~A}$ and $1 \mathrm{~B})$. Western blot assays showed consistent outcomes with those based on RT-qPCR experiments (Figure 1C and 1D).

\subsection{MMP2 knockdown decreased cellular proliferation and increased cellular apoptosis}

To investigate the biological functions of MMP2 in HCC, we blocked MMP2 expression in HepG2 and Huh7 cells by si-MMP2, and cell proliferation and apoptosis were then assessed using MTT assays and flow cytometry analysis, respectively. Optical density (OD) values from the MTT assays indicate that, compared with that of the normal control cells, the proliferation of HepG2 and Huh7 cells infected with si-MMP2 lentivirus was significantly inhibited; however, the proliferation of cells from the remaining three groups did not significantly differ from that of the normal control cells (Figure 2).

Annexin V/PI double staining and flow cytometry analysis were performed to further evaluate the apoptosis of HepG2 and Huh7 cells after MMP2 knockdown (Figure 3). In both cell lines, no significant differences in the apoptotic rate were observed among the control, polybrene, and si-con groups in both cell lines ( $\mathrm{P}>0.05)$. However, compared with that in any other group from the same cell line, apoptosis in the si-MMP2 group significantly increased $(\mathrm{P}<0.01)$.

\subsection{MMP2 knockdown inhibited the cell invasive capacity of HCC cells}

To further determine whether MMP2 was involved in the invasion of HCC cells, Transwell assays were performed (Table 1; Supplementary Figure 3). The invasive cell counts of the control, polybrene, and si-con groups were comparable to that reported in the literature [20]. In both cell lines, no significant differences in the number of invasive cells were observed among the $\mathrm{NC}$, polybrene, and si-con groups $(\mathrm{P}>0.05)$. However, compared with that in any other group from the same cell line, the number of invasive cells in the si-MMP2 group significantly decreased $(\mathrm{P}<0.01)$.

\subsection{MMP2 knockdown increased the sensi- tivity of HCC cells to cisplatin}

HCC cells were treated with si-MMP2 and cisplatin (50 $\mu \mathrm{g} / \mathrm{ml}$ ), and an MTT assay was then performed to assess cell viability (Figure 4). No significant differences in the OD values were observed among the $\mathrm{NC}+$ cisplatin, polybrene+cisplatin, and si-con+cisplatin groups in both cell lines $(\mathrm{P}>0.05)$. However, compared with that in any other group from the same cell line, the OD value in the si-MMP2+cisplatin group significantly decreased $(\mathrm{P}<0.01)$.

\section{Discussion}

In this study, we demonstrated that knockdown of MMP2 in HepG2 and Huh7 cell lines by infecting cells with si-MMP2 lentivirus strongly inhibited cell proliferation and invasion and significantly promoted cell proliferation.

The process of invasion and migration of tumour cells requires degradation of the ECM and basement membrane, and MMPs that are expressed by almost all tumour cells perform this proteolytic process [21-23]. According to their exact functions and distributions in cells, MMPs can be divided into multiple subtypes. MMP2 is an important member of the MMP family that was found to be overexpressed in multiple human tumours, including HCC [24, 25]. Liu et al. [26] demonstrated that downregulation of MMP2 and MMP9 was induced by the main components of pomegranate, ellagic acid and luteolin, and suppressed the metastasis of ovarian cancer. Downregulation of MMP2 and MMP9 induced by TRIM31 knockdown was shown to suppress the proliferation and invasion of gallbladder cancer [27]. In addition, Fang et al. [28] reported 

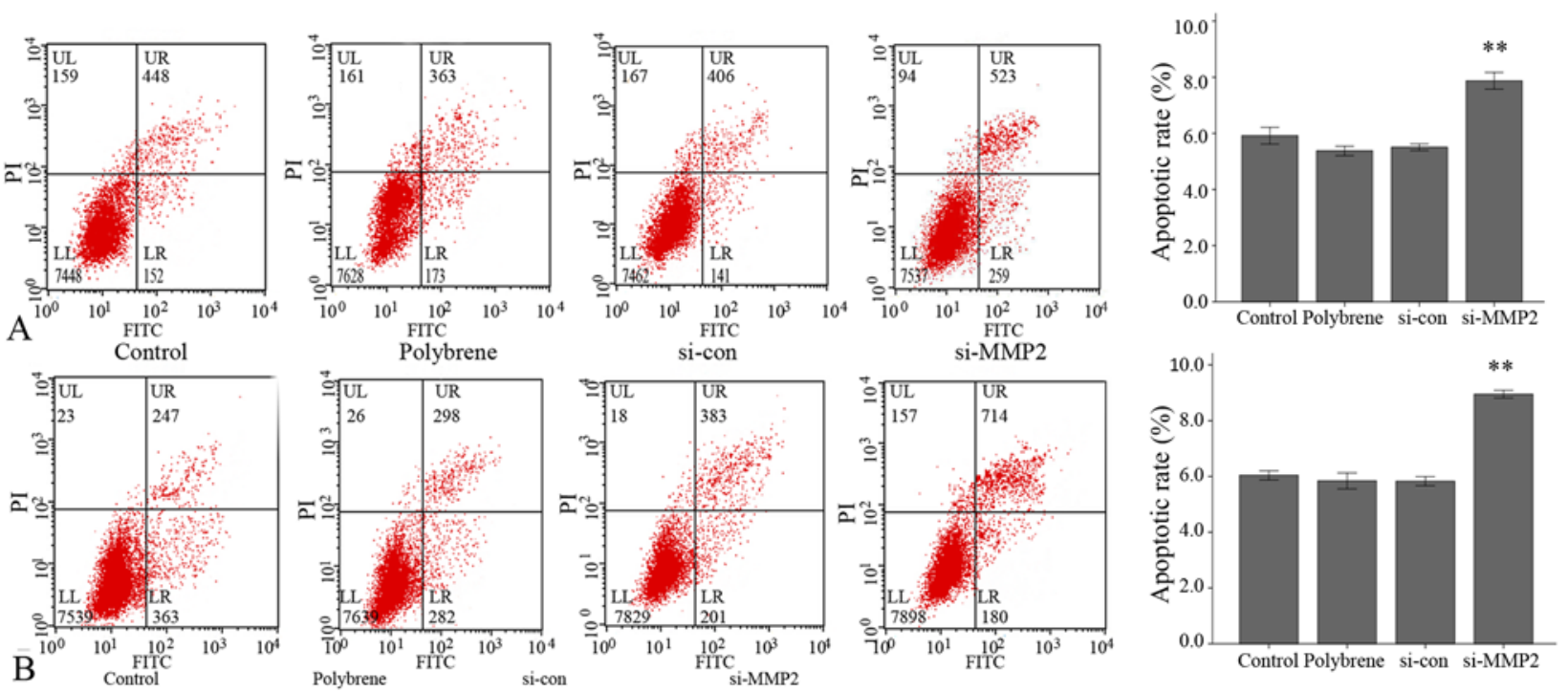

Figure 3. The apoptotic rates of HepG2 (A; at $48 \mathrm{~h}$ after transduction) and Huh7 cells (B; at $72 \mathrm{~h}$ after transduction) using flow cytometry. The numbers in the graphs are the counts of the cells detected with flow cytometry (UL, necrotic cells; LL, non-apoptotic cells; LR, cells at the early apoptotic stage; and UR, cells at the late apoptotic stage). The apoptotic rate $=$ the apoptotic rate at the early stage (LR) + that at the late stage (UR). ${ }^{* *} \mathrm{P}<0.01 \mathrm{vs}$. the normal control group, according to one-factor analysis of variance (ANOVA) followed by a post hoc least significant difference (LSD) test.

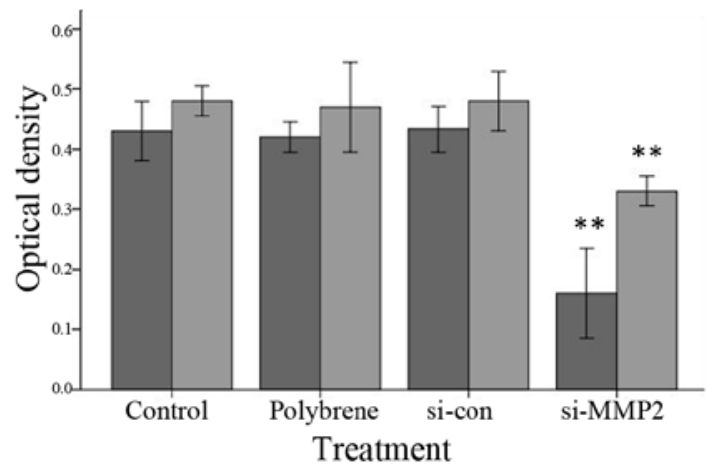

Figure 4. Effects of MMP2 knockdown on the sensitivity HepG2 (at $48 \mathrm{~h}$ after transduction) and Huh7 cells (at $72 \mathrm{~h}$ after transduction) to cisplatin using MTT assays. ${ }^{\star *} \mathrm{P}<0.01$ vs. the normal control group, according to one-factor analysis of variance (ANOVA) followed by a post hoc least significant difference (LSD) test.

that downregulation of MMP2 induced by microRNA-29b overexpression significantly inhibited tumour angiogenesis, invasion, and metastasis of HCC. Our results were consistent with previously reported results [28].

Given the complexity of the pathogenesis of HCC, the current therapeutic options for HCC mainly include surgical resection, liver transplantation, and chemotherapy with several antitumour drugs, such as cisplatin, sorafenib, and brivanib $[29,30]$. Currently, cisplatin remains the first-line chemotherapeutic agent for HCC [31]. In this study, cisplatin was applied to HCC cells after MMP2 knockdown. The results showed that, compared with those of the other groups, the OD values of the MMP2 knockdown groups were substantially decreased, suggest-
Table 1: Invasive cell counts of HepG2 and Huh7 cells after lentivirus-mediated transduction (at $48 \mathrm{~h}$ and $72 \mathrm{~h}$, respectively) based on Transwell assays.

\begin{tabular}{lll}
\hline & HepG2 & Huh7 \\
\hline Normal control & $276 \pm 15$ & $136 \pm 22$ \\
Polybrene & $213 \pm 11$ & $139 \pm 9$ \\
si-con & $231 \pm 24$ & $145 \pm 21$ \\
si-MMP2 & $126 \pm 15^{\star \star}$ & $76 \pm 8^{\star \star}$ \\
\hline
\end{tabular}

** $P<0.01$ vs. the normal control group, according to one-factor analysis of variance (ANOVA) followed by a post hoc least significant difference (LSD) test.

ing that MMP2 knockdown may have a synergistic reaction with cisplatin in inhibiting the proliferation of HCC cells. Recently, an inhibitor of MMP2 was shown to enhance the sensitivity of platinum-resistant ovarian cancer cells to cisplatin [32]. In addition, Li et al. reported that genistein could reinforce the inhibitory effects of cisplatin on HCC by downregulating MMP2 expression [33]. Our results are consistent with those previously reported, suggesting that MMP2 may serve as a target in the treatment of HCC in clinical practice.

This study has limitations. This study was conducted in vitro, and therefore, microenvironmental changes in vivo may alter the results. Furthermore, in this study, two HCC cell lines were employed. To further validate the roles of MMP2 in the proliferation and invasion of HCC cells, more cell lines and in vivo studies should be involved in 
future studies. In conclusion, MMP2 knockdown inhibits the progression of HepG2 and Huh7 cells and enhances the cytotoxicity of cisplatin to these cells, suggesting that MMP2 may act as a therapeutic target for HCC patients.

Acknowledgements: This work was supported by the Guizhou Province Science and Technology Fund Project; (No. J (Altekruse, et al.) 2113) and the Joint Fund Project of Guizhou Science and Technology Department (grant number, [2014]2113).

Conflicts of interest: The authors have no commercial or other associations that might pose a conflict of interest.

\section{References}

[1] Ban, D., Ogura, T., Akahoshi, K., Tanabe, M., Current topics in the surgical treatments for hepatocellular carcinoma, Ann. Gastroenterol. Surg., 2018, 2, 137-146

[2] Yen, C.W., Hsu, L.S., Chen, C.W., Lin, W.H., Hepatocellular carcinoma with thoracic metastases presenting as hemothorax: A case report and literature review, Medicine (Baltimore), 2018, 97, e10945

[3] Kamo, N., Kaido, T., Yagi, S., Okajima, H., Uemoto, S., Liver transplantation for intermediate-stage hepatocellular carcinoma, Liver Cancer, 2018, 7, 179-189

[4] Niu, L.Z., Li, J.L., Zeng, J.Y., Mu, F., Liao, M.T., Yao, F., et al., Combination treatment with comprehensive cryoablation and immunotherapy in metastatic hepatocellular cancer, World J. Gastroenterol., 2013, 19, 3473-3480

[5] Schlachterman, A., Craft, W.W. Jr., Hilgenfeldt, E., Mitra, A., Cabrera, R., Current and future treatments for hepatocellular carcinoma, World J. Gastroenterol., 2015, 21, 8478-8491

[6] Egeblad, M., Werb, Z., 2002 New functions for the matrix metalloproteinases in cancer progression, Nat. Rev. Cancer, 2002, 2, 161-174

[7] Sternlicht, M.D., Werb, Z., How matrix metalloproteinases regulate cell behavior, Annu. Rev. Cell Dev. Biol., 2001, 17, 463-516

[8] Lohi, J., Wilson, C.L., Roby, J.D., Parks, W.C., Epilysin, a novel human matrix metalloproteinase (MMP-28) expressed in testis and keratinocytes and in response to injury, J. Biol. Chem., 2001, 276, 10134-10144

[9] Herrera, I., Cisneros, J., Maldonado, M., Ramírez, R., Ortiz-Quintero, B., Anso, E., et al., Matrix metalloproteinase (MMP)-1 induces lung alveolar epithelial cell migration and proliferation, protects from apoptosis, and represses mitochondrial oxygen consumption, J. Biol. Chem., 2013, 288, 25964-25975

[10] Kraljevic, D., Vukojevic, K., Karan, D., Rajic, B., Todorovic, J., Miskovic, J., et al., Proliferation, apoptosis and expression of matrix metalloproteinase-9 in human fetal lung, Acta Histochem, 2015, 117, 444-450.
[11] Kalhori, V., Tornquist, K., MMP2 and MMP9 participate in S1P-induced invasion of follicular ML-1 thyroid cancer cells, Mol. Cell Endocrinol., 2015, 404, 113-122

[12] Laios, A., Mohamed, B.M., Kelly, L., Flavin, R., Finn, S., McEvoy, L., et al., Pre-treatment of platinum resistant ovarian cancer cells with an MMP-9/MMP-2 inhibitor prior to cisplatin enhances cytotoxicity as determined by high content screening, Int. J. Mol. Sci., 2013, 14, 2085-2103

[13] Liu, J., Ping, W., Zu, Y., Sun, W., Correlations of lysyl oxidase with MMP2/MMP9 expression and its prognostic value in non-small cell lung cancer, Int. J. Clin. Exp. Pathol., 2014, 7, 6040-6047

[14] Maacha, S., Hong, J., von Lersner, A., Zijlstra, A., Belkhiri, A., AXL mediates esophageal adenocarcinoma cell invasion through regulation of extracellular acidification and lysosome trafficking. Neoplasia, 2018, 20, 1008-1022

[15] Ni, X., Xia, T., Zhao, Y., Zhou, W., Wu, N., Liu, X., et al., Downregulation of miR-106b induced breast cancer cell invasion and motility in association with overexpression of matrix metalloproteinase 2. Cancer Sci., 2014, 105, 18-25

[16] Zhang, C., Wang, G., Wang, J., Ji, Z., Dong, F., Chao, T., Analysis of differential gene expression and novel transcript units of ovine muscle transcriptomes. PLoS One, 2014, 9, e89817

[17] Saha, P., Ghosh, I., Datta, K., Increased hyaluronan levels in $\mathrm{HABP} 1 / \mathrm{p} 32 / \mathrm{gC} 1 \mathrm{qR}$ overexpressing HepG2 cells inhibit autophagic vacuolation regulating tumor potency. PLoS One, 2014, 9, e103208

[18] Khantasup, K., Kopermsub, P., Chaichoun, K., Dharakul, T., Targeted small interfering RNA-immunoliposomes as a promising therapeutic agent against highly pathogenic Avian Influenza A (H5N1) virus infection. Antimicrob Agents Chemother, 2014, 58, 2816-2824

[19] Kim, M., Jung, J.Y., Choi, S., Lee, H., Morales, L.D., Koh, J.T., et al., GFRA1 promotes cisplatin-induced chemoresistance in osteosarcoma by inducing autophagy. Autophagy, 2017, 13, 149-168

[20] Mao, H.H., In vitro experimental study on ant-FAK short hairpin RNA for metastasis of colorectal cancer. Dissertation. Fujian Medical University, 2010

[21] Chojnacki, M., Zajac, A., Piet, M., The involvement of matrix metalloproteinases in the development and progression of neoplasm diseases, Postepy. Biochem., 2017, 63, 277-286

[22] Das, K., Prasad, R., Ansari, S.A., Roy, A., Mukherjee, A., Sen, P., Matrix metalloproteinase-2: A key regulator in coagulation proteases mediated human breast cancer progression through autocrine signaling, Biomed. Pharmacother., 2018, 105, 395-406

[23] Yadav, L., Puri, N., Rastogi, V., Satpute, P., Ahmad, R., Kaur, G., Matrix metalloproteinases and cancer-roles in threat and therapy, Asian Pac. J. Cancer Prev., 2014, 15, 1085-1091

[24] Giannelli, G., Bergamini, C., Marinosci, F., Fransvea, E., Quaranta, M., Lupo, L., et al., Clinical role of MMP-2/ TIMP-2 imbalance in hepatocellular carcinoma, Int. J. Cancer, 2002, 97, 425-431

[25] Ogasawara, S., Yano, H., Momosaki, S., Nishida, N., Takemoto, Y., Kojiro, S., et al., Expression of matrix metalloproteinases (MMPs) in cultured hepatocellular carcinoma (HCC) cells and surgically resected HCC tissues, Oncol. Rep., 2005, 13, 1043-1048 
[26] Liu, H., Zeng, Z., Wang, S., Li, T., Mastriani, E., Li, Q.H., et al., Main components of pomegranate, ellagic acid and luteolin, inhibit metastasis of ovarian cancer by down-regulating MMP2 and MMP9, Cancer Biol. Ther., 2017, 18, 990-999

[27] Li, H., Zhang, Y., Hai, J., Wang, J., Zhao, B., Du, L., et al., Knockdown of TRIM31 suppresses proliferation and invasion of gallbladder cancer cells by down-regulating MMP2/9 through the PI3K/Akt signaling pathway, Biomed. Pharmacother., 2018, 103, 1272-1278

[28] Fang, J.H., Zhou, H.C., Zeng, C., Yang, J., Liu, Y., Huang, X., et al., MicroRNA-29b suppresses tumor angiogenesis, invasion, and metastasis by regulating matrix metalloproteinase 2 expression, Hepatology, 2011, 54, 1729-1740

[29] Hatzaras, I., Bischof, D.A., Fahy, B., Cosgrove, D., Pawlik, T.M., Treatment options and surveillance strategies after therapy for hepatocellular carcinoma, Ann. Surg. Oncol., 2014, 21, 758-766

[30] Raza, A., Sood, G.K., Hepatocellular carcinoma review: current treatment, and evidence-based medicine, World J. Gastroenterol., 2014, 20, 4115-4127

[31] Dasari, S., Tchounwou, P.B., Cisplatin in cancer therapy: molecular mechanisms of action, Eur. J. Pharmacol., 2014, 740, 364-378

[32] Nouso, K., Miyahara, K., Uchida, D., Kuwaki, K., Izumi, N., Omata, M., et al., Effect of hepatic arterial infusion chemotherapy of 5 -fluorouracil and cisplatin for advanced hepatocellular carcinoma in the Nationwide Survey of Primary Liver Cancer in Japan, Br. J. Cancer, 2013, 109, 1904-1907

[33] Chen, P., Hu, M.D., Deng, X.F., Li, B., Genistein reinforces the inhibitory effect of Cisplatin on liver cancer recurrence and metastasis after curative hepatectomy, Asian Pac. J. Cancer Prev., 2013, 14, 759-764 